\title{
An innovative Method to Extend the Service Life of Future Infrastructure Systems by Using Alternative Materials
}

\author{
Maziar Mahdavi* and Arash Emami Saleh \\ Post Doctoral Research Associate, The University of Texas at Arlington, Arlington, Texas, USA
}

*Corresponding author: Maziar Mahdavi, Post Doctoral Research Associate, The

University of Texas at Arlington, Arlington, Texas, USA.

Received Date: April 18, 2020

Published Date: April 28, 2020

\section{Abstract}

Infrastructures maintenance has always been a very expensive and sophisticated part of the governments' asset management worldwide. Among these infrastructure systems, pipelines and specifically concrete pipes are very important and extending their service life would highly benefit governments around the world. This article explores different aspects of a novel material which can improve the durability of these important infrastructure systems.

\section{Introduction}

Infrastructures are one of the vital piers of a stable economy. Construction, maintenance and reconstruction of infrastructure systems are highly expensive and extending their service life is one of the biggest engineering challenges that faces governments and private sectors worldwide. Plenty of research has been conducted in the field of infrastructure's service life and each of those has focused on a specific aspect of this challenge. Among these infrastructure systems, pipelines are highly important in different engineering applications, and specifically reinforced concrete pipes have been widely used in water and wastewater systems. Reinforced concrete pipes are inherently susceptible to environmental deterioration because of the embedded steel reinforcements. For example, in sewer lines or in pipes that are used in coastal regions, steel reinforcement would get corroded because of acid or chlorideinduced corrosion. In this article, recent advances in proposing a novel approach to eliminate these undesirable phenomena will be reviewed.

\section{Synthetic Fiber Reinforced Concrete Pipes}

Traditionally, active and passive corrosion control methods have been used to preserve embedded steel reinforcements. Besides, eliminating steel and substituting them with alternative reinforcing elements has been investigated by many researchers. Researchers at the University of Texas at Arlington developed a very promising synthetic fiber reinforced concrete (SYNFRC) material to be used in concrete pipe systems. Wilson \& Abolmaali [1] have used the fibers that are shown in Figure 1 as an alternative to steel reinforcement in concrete pipes in different fiber volume fractions.

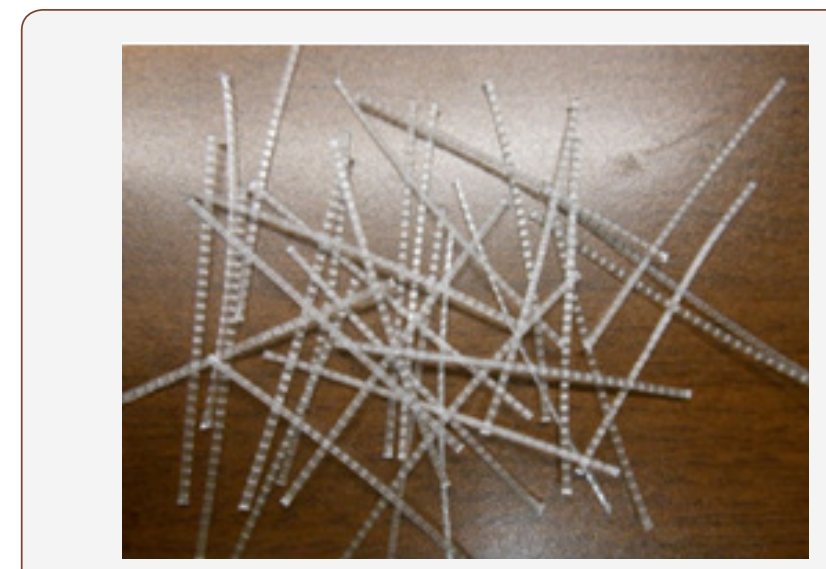

Figure 1: BASF MasterFiber MAC Matrix macro synthetic fibers.

The volume fractions (Vf) utilized in the study were 0.26 , $0.39,0.52,0.65,0.78,1.04$, and 1.17 percent and93 SYNFRCP with diameters from 15-in to 36-in. were produced in three different sites and locations to ensure the variety of the equipment and geographical locations. 
In higher Vfs the ultimate load has decreased with increasing the amount of fibers.

After the initial evaluation of this new reinforced concrete, since the reinforcing material is plastic and sustained loading of plastic would be a source of concern, researchers at the University of Texas at Arlington investigated the time-dependent behavior of this new material under sustained load. Park et al. [2] performed long-term tests on buried SYNFRCPs with two different fiber dosages. The fiber dosages were $8 \mathrm{lb} . / \mathrm{yd} 3\left(4.8 \mathrm{~kg} / \mathrm{m}^{3}\right)$ and $12 \mathrm{lb} . /$ $\operatorname{yd} 3\left(7.0 \mathrm{~kg} / \mathrm{m}^{3}\right)$ and the diameters of the tested pipes were 24-in and 36-in.In addition to SYNFRCPs, RCPs were also tested under the same loading conditions to compare the long-term performance of this new material with the ordinary reinforced concrete pipes. In addition, test pipes were precracked to determine the behavior of these fibers in controlling crack propagation. The sustained load in this test setup was $1,350 \mathrm{lb} . / \mathrm{ft} / \mathrm{ft}(65 \mathrm{kN} / \mathrm{m} / \mathrm{m})$ based on ASTM C76 and their deflections were recorded with a series of displacement transducers. After 4200 hours of testing, as shown in Figure 2, it was determined that SYNFRCPs experienced $2.83 \%$ and $1.61 \%$ deflection in 24-in. and 36-in. pipes, respectively and the fibers held the cracks and prevented the pipes from exceeding 3\% deflection. These results showed that the time-dependent behavior of pipes was not deteriorated by utilizing synthetic fiber reinforcements.
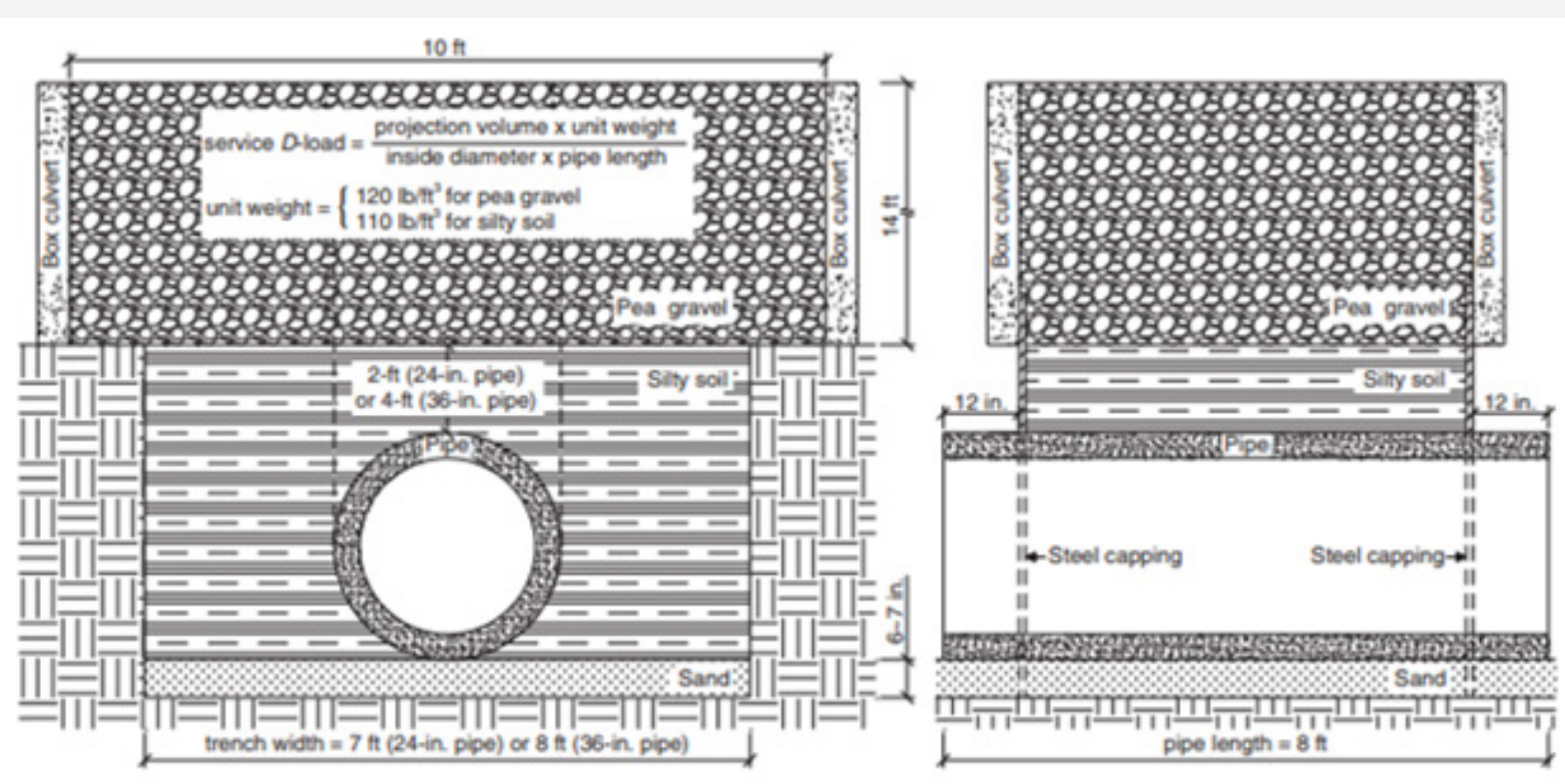

Figure 2: Sustained load test setup.



Figure 3: SYNFRC beam shear test [3].

As the next step in verifying the behavior of this novel composite in different loading conditions, Mostafazadeh \& Abolmaali [3] studied the behavior of SYNFRC beams under shear loading scenarios. For this purpose, two different compressive strengths, $28 \mathrm{MPa}$ (4000 psi) and $34 \mathrm{MPa}$ (5000 psi) were chosen and four different fiber volume fractions were utilized. For each of these cases three $150 \times 150 \times 500 \mathrm{~mm}(6 \times 6 \times 20 \mathrm{in}$. $)$ beams were produced and they were tested based on ASTM C1609, as depicted in Figure 3. The results showed that adding these fibers to concrete mixes increases the shear toughness of the sections by $648 \%$ and $736 \%$ for $28 \mathrm{MPa}$ (4000 psi) and $34 \mathrm{MPa}$ (5000 psi) mixes. Besides, further investigations were conducted by Ghahremannejad et al. [5] 
to address the crack width and crack distribution in SYNFRC beams with single and multiple crack scenarios. Digital Image Correlation (DIC) measurement method was utilized in this study to accurately measure the crack width in these beams. The results showed that using higher fiber volume fractions leads to higher load bearing capacities by increasing the bridging action of the fibers. It was also determined that higher fiber dosages would result in improved serviceability which is vital in infrastructure systems.

According to these studies, it was shown that SYNFRCPs can be safely used as an alternative to conventional reinforced concrete pipes based on the existing load bearing criteria. On the other hand, one of the main concerns after proposing a new infrastructure material would be its durability, which was the main concern

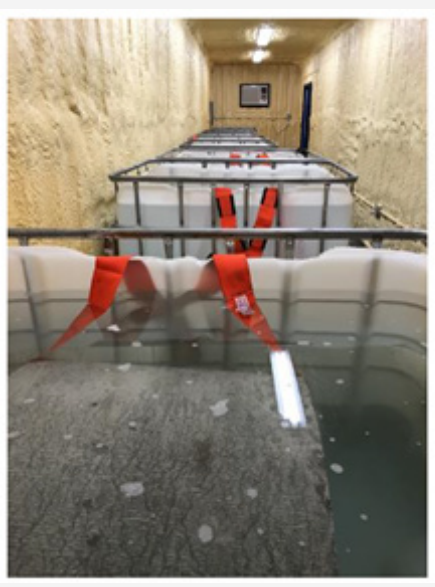

Figure 4: Service life prediction test setup [5].

\section{Conclusion}

According to the reviewed studies in this article, and considering the relatively short service life of conventional RCPs, SYNFRCPs can be reliably used instead of the traditional pipes whenever the productions pass the tests and meet the requirements of the standards proposed for this specific composite.

\section{Acknowledgement}

None.

\section{Conflict of Interest}

No conflict of interest.

\section{References}

1. Wilson A, Abolmaali A (2014) Performance of synthetic fiber-reinforced concrete pipes. Journal of Pipeline Systems Engineering and Practice 5(3): 04014002. that led to the development of this new composite. To study the durability of this composite, Mahdavi et al. [5], has developed a test protocol to accelerate the deterioration scenarios of sewer and drainage pipes by decreasing the $\mathrm{pH}$ of the fluid that affect the pipe and increasing the ambient temperature. Both measures would accelerate the deterioration reactions that affect the durability of the pipe material. After a year-long test, as shown in Figure 4, it was concluded that SYNFRCPs can survive in the harsh field environments for 375 years while conventional RCPs cannot last longer than 50 years in these conditions. It should be noted that the service life requirement for major pipelines that is usually mandated to be 100 years by the Departments of Transportation and Mahdavi [6] showed that the new composite can improve the shortcomings of the traditional materials.

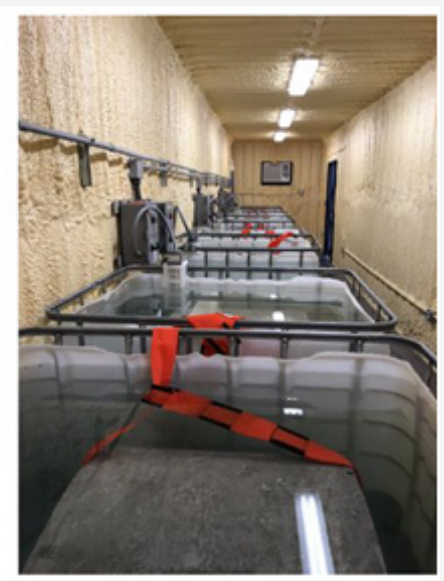

2. Park Y, Abolmaali A, Attiogbe E, Lee SH (2014) Time-Dependent Behavior of Synthetic Fiber-Reinforced Concrete Pipes Under LongTerm Sustained Loading. Transportation Research Record 2407(1): 7179.

3. Mostafazadeh M, Abolmaali A (2016) Shear behavior of synthetic fiber reinforced concrete. Advances in Civil Engineering Materials 5(1): 371386.

4. Ghahremannejad M, Mahdavi M, Saleh AE, Abhaee S, Abolmaali A (2018) Experimental investigation and identification of single and multiple cracks in synthetic fiber concrete beams. Case Studies in Construction Materials 9: e00182.

5. Mahdavi M (2019) Development of Protocol for 100-Year Service Life of Synthetic Fiber-Reinforced Concrete Pipes (Doctoral dissertation).

6. Mahdavi M, Abolmaali A, Ghahremannejad M (2018) The Effects of $\mathrm{pH}$ and Temperature on Compressive Strength of Synthetic FiberReinforced Concrete Cylinders Exposed to Sulfuric Acid. Advances in Civil Engineering Materials 7(1): 403-413. 